\title{
THE PREVALENCE OF CONTINUOUS NOWHERE DIFFERENTIABLE FUNCTIONS
}

\author{
BRIAN R. HUNT
}

(Communicated by Andrew M. Bruckner)

\begin{abstract}
In the space of continuous functions of a real variable, the set of nowhere differentiable functions has long been known to be topologically "generic". In this paper it is shown further that in a measure theoretic sense (which is different from Wiener measure), "almost every" continuous function is nowhere differentiable. Similar results concerning other types of regularity, such as Hölder continuity, are discussed.
\end{abstract}

\section{INTRODUCTION}

In [5] a translation-invariant, measure-theoretic definition was given for the notion of "almost every" on infinite-dimensional spaces. ${ }^{1}$ This concept, called "prevalence", is intended to replace the topological notion of "genericity" in contexts where a measure-theoretic result is preferred. The goal of this paper is to show that in terms of prevalence, almost every continuous function of a real variable is nowhere differentiable. ${ }^{2}$

We denote by $C[0,1]$ the Banach space of continuous functions from $[0,1]$ to $\mathbb{R}$. For simplicity we consider only functions in this space, though our results also hold for functions defined on all of $\mathbb{R}$. Our main result is as follows.

Theorem 1. Almost every function in $C[0,1]$ is nowhere differentiable; that is, the nowhere differentiable functions form a prevalent subset of $C[0,1]$.

Before we discuss the general definition of prevalence, let us state in more familiar terms exactly what we will prove about the set of nowhere differentiable functions in $C[0,1]$.

Proposition 1. There exist functions $g$ and $h$ in $C[0,1]$ such that for all $f \in$ $C[0,1]$, the function $f+\lambda g+\mu h$ is nowhere differentiable for Lebesgue almost every $(\lambda, \mu) \in \mathbb{R}^{2}$.

Received by the editors November 11, 1992.

1991 Mathematics Subject Classification. Primary 26A27, 26A21; Secondary 28C20, 60B11.

The author was supported by the National Science Foundation (Divisions of Mathematical and Physical Sciences).

${ }^{1}$ After [5] went to press, it was discovered that Christensen [3] gave an analogous definition of a "Haar zero set" on abelian Polish groups; see [6].

${ }^{2}$ By "differentiable" we will always mean "having a finite derivative".

(C) 1994 American Mathematical Society $0002-9939 / 94 \$ 1.00+\$ .25$ per page 
In other words, the space $C[0,1]$ can be partitioned into parallel planes in such a way that in each plane, almost every function (with respect to Lebesgue measure) is nowhere differentiable. The plane spanned by $g$ and $h$ is then a "probe" for the set of nowhere differentiable functions in the sense of the following definition.

Definition 1. Given a set $S \subset C[0,1]$, we say that a finite-dimensional subspace $P \subset C[0,1]$ is a probe for $S$ provided that for all $f \in C[0,1]$, Lebesgue almost every point in the hyperplane $f+P$ belongs to $S$.

If a Borel set $S \subset C[0,1]$ has a probe, then we say $S$ is prevalent. (However, a Borel set can be prevalent without having a probe; see [5] for the precise definition of prevalence.) A non-Borel set is prevalent if it contains a prevalent Borel set.

Thus Proposition 1 would directly imply Theorem 1, with a two-dimensional probe, were it not for the fact that the set of nowhere differentiable functions is not a Borel subset of $C[0,1][9,10]$. However, this set contains the set of nowhere Lipschitz functions (that is, functions which have no finite Lipschitz constant at any point), and we will show that the latter set is both Borel and prevalent, using the same two-dimensional probe.

Though we do not work out the details in this paper, using higher-dimensional probes we can further show that almost every function in $C[0,1]$ is nowhere Hölder continuous. This is a good example of the difference between using prevalence and using Wiener measure to define "almost every" on $C[0,1]$; with respect to Wiener measure, almost every function in $C[0,1]$ is in the Hölder class $C^{\gamma}$ for every $\gamma<1 / 2$.

Another way in which "almost every" results have been formulated on function spaces like $C[0,1]$ is in terms of the topological notion of "genericity". A property on a complete metric space is said to be generic if the set on which it holds contains a countable intersection of open dense sets. The complement of such a set is said to be of the first category; equivalently, a first category set is a countable union of nowhere dense sets. The Baire category theorem (see [12]) ensures that a generic property holds on a dense subset of the space.

In $\mathbb{R}^{n}$ a property can be topologically generic but have zero probability, in the sense that it holds only on a set of Lebesgue measure zero. Likewise a first category set can contain Lebesgue almost every point in the space. Furthermore, these kinds of sets can arise naturally; several examples are discussed in [5]. The notion of prevalence is equivalent to "Lebesgue almost every" in $\mathbb{R}^{n}$.

It has long been known that the nowhere differentiable functions are topologically generic in $C[0,1]$. This result was proved originally by Banach [2] and Mazurkiewicz [11] (see also §III.34.VIII of [8] or $\S 11$ of [12] for a proof, and for a brief explanation see the beginning of $\S 3$ of this paper). More recently the set of nowhere differentiable functions in $C[0,1]$ has been shown to be "large" in the stronger sense of "porosity" (see [13] and the references therein).

In $\S 2$ we construct the functions $g$ and $h$ which span our probe space, and obtain an estimate on the irregularity of the functions in the probe space. We use this estimate in $\S 3$ to prove Theorem 1 and Proposition 1 , and in $\S 4$ we briefly indicate some ways in which our main result can be extended. 


\section{THE PROBE SPACE}

Let us first explain why there is no one-dimensional probe space for the set of continuous nowhere differentiable functions. Consider the space spanned by a function $g \in C[0,1]$. Let $f(x)=-x g(x)$, and notice that $f+\lambda g$ is differentiable at $x=\lambda$ for every $\lambda \in[0,1]$. Thus for a set of $\lambda$ with positive Lebesgue measure, $f+\lambda g$ is not nowhere differentiable.

The functions $g$ and $h$ which span our probe space are based on the famous Weierstrass nowhere differentiable function, given by

$$
f(x)=\sum_{k=0}^{\infty} a^{k} \cos b^{k} \pi x
$$

where $1 \leq a b<b$. Notice that $f$ is continuous because the sum converges uniformly. Weierstrass proved that this function is nowhere differentiable for some of these values of $a$ and $b$ (see [4]), while Hardy [4] gave the first proof for all such $a$ and $b$. An elementary proof of this result can be constructed in much the same way as the proof of Lemma 1 below.

Let

$$
g(x)=\sum_{k=1}^{\infty} \frac{1}{k^{2}} \cos 2^{k} \pi x, \quad h(x)=\sum_{k=1}^{\infty} \frac{1}{k^{2}} \sin 2^{k} \pi x .
$$

The function $g$ was proposed by Hardy [4] as an example of an absolutely continuous Fourier series whose sum is nowhere Hölder continuous. This result follows from the next lemma, which says that on intervals of length $2^{-k}$, functions in the space spanned by $g$ and $h$ must have fluctuations of magnitude at least proportional to $1 / k^{2}$.

Lemma 1. There exists a constant $c>0$ such that for any $\alpha, \beta \in \mathbb{R}$ and any closed interval $I \subset[0,1]$ with length $\varepsilon \leq 1 / 2$,

$$
\max _{I}(\alpha g+\beta h)-\min _{I}(\alpha g+\beta h) \geq \frac{c \sqrt{\alpha^{2}+\beta^{2}}}{(\log \varepsilon)^{2}} .
$$

Remark. The proof of this lemma is based on a similar argument by Kaplan, Mallet-Paret, and Yorke [7] which is used in determining the box-counting dimension of the graph of the Weierstrass nowhere differentiable function. Incidentally, Lemma 1 implies that the graphs of $g$ and $h$ have box-counting dimension 2.

Proof. Let $I$ be a closed interval in $[0,1]$ of length $2^{-m}$ for some positive integer $m$. We claim that for any continuous $f$,

$$
\max _{I} f-\min _{I} f \geq 2^{m} \pi \int_{I} f(x) \cos \left(2^{m+j} \pi x+\theta\right) d x
$$

for all positive integers $j$ and all $\theta \in[0,2 \pi)$. Since $\cos \left(2^{m+j} \pi x+\theta\right)$ has integral zero over $I$, adding a constant to $f$ does not alter either side of (3). Thus we may assume that $\max _{I} f=-\min _{I} f=K$ for some $K \geq 0$. Then $|f| \leq K$ on $I$, and hence

$$
2^{m} \pi \int_{I} f(x) \cos \left(2^{m+j} \pi x+\theta\right) d x \leq 2^{m} \pi K \int_{I}\left|\cos \left(2^{m+j} \pi x+\theta\right)\right| d x .
$$


Since the length of $I$ is a multiple of the period of $\left|\cos \left(2^{m+j} \pi x+\theta\right)\right|$, the integral of this function is equal to the length of $I$, which is $2^{-m}$, times the average value of the function, which is $2 / \pi$. Thus

$$
2^{m} \pi \int_{I} f(x) \cos \left(2^{m+j} \pi x+\theta\right) d x \leq 2^{m} \pi K 2^{-m} \frac{2}{\pi}=2 K,
$$

which is equivalent to (3).

Now let $f=\alpha g+\beta h$; then

$$
f(x)=\sum_{k=1}^{\infty} \frac{1}{k^{2}}\left(\alpha \cos 2^{k} \pi x+\beta \sin 2^{k} \pi x\right)=\sqrt{\alpha^{2}+\beta^{2}} \sum_{k=1}^{\infty} \frac{1}{k^{2}} \cos \left(2^{k} \pi x+\theta\right),
$$

where $\theta \in[0,2 \pi)$ depends only on $\alpha$ and $\beta$. By replacing $f$ with the function $f / \sqrt{\alpha^{2}+\beta^{2}}$ we may assume that $\alpha^{2}+\beta^{2}=1$. Then by (3), for all positive integers $j$,

$$
\begin{aligned}
\max _{I} f-\min _{I} f \geq 2^{m} \pi \int_{I} \sum_{k=1}^{\infty} \frac{1}{k^{2}} \cos \left(2^{k} \pi x+\theta\right) \cos \left(2^{m+j} \pi x+\theta\right) d x \\
=\sum_{k=1}^{\infty} \frac{2^{m} \pi}{2 k^{2}} \int_{I}\left(\cos \left(\left(2^{m+j}-2^{k}\right) \pi x\right)+\cos \left(\left(2^{m+j}+2^{k}\right) \pi x+2 \theta\right)\right) d x
\end{aligned}
$$

Each of the functions to be integrated has the form $\cos \left(\left(2^{m+j}+\omega\right) \pi x+\varphi\right)$ where $\omega= \pm 2^{k}$ and $\varphi$ is either 0 or $2 \theta$. If $k>m$, then $2^{m+j}+\omega$ is a multiple of $2^{m+1}$. Since $I$ has length $2^{-m}$, it follows that $\cos \left(\left(2^{m+j}+\omega\right) \pi x+\varphi\right)$ has integral zero over $I$ for $k>m$, unless $\omega=-2^{m+j}$, in which case $\varphi=0$ and the integrand is identically one, so its integral over $I$ is $2^{-m}$. For $k \leq m$, we use the following estimate in which $y$ denotes the left endpoint of $I$ :

$$
\begin{aligned}
\int_{I} \cos \left(\left(2^{m+j}+\omega\right) \pi x+\varphi\right) d x \\
\quad=\frac{\sin \left(\left(2^{m+j}+\omega\right) \pi\left(y+2^{-m}\right)+\varphi\right)-\sin \left(\left(2^{m+j}+\omega\right) \pi y+\varphi\right)}{\left(2^{m+j}+\omega\right) \pi} \\
\quad=\frac{\sin \left(\left(2^{m+j}+\omega\right) \pi y+\varphi+2^{-m} \pi \omega\right)-\sin \left(\left(2^{m+j}+\omega\right) \pi y+\varphi\right)}{\left(2^{m+j}+\omega\right) \pi} \\
\quad \geq-\frac{\left|2^{-m} \pi \omega\right|}{\left(2^{m+j}+\omega\right) \pi}=-\frac{|\omega|}{2^{m}\left(2^{m+j}+\omega\right)} .
\end{aligned}
$$

It then follows from the previous paragraph that

$$
\begin{aligned}
\max _{I} f-\min _{I} f & \geq \frac{\pi}{2(m+j)^{2}}-\sum_{k=1}^{m} \frac{\pi}{2 k^{2}}\left(\frac{2^{k}}{2^{m+j}-2^{k}}+\frac{2^{k}}{2^{m+j}+2^{k}}\right) \\
& \geq \frac{\pi}{2(m+j)^{2}}-\left(\sum_{k=1}^{m} \frac{2^{k}}{k^{2}}\right) \frac{\pi}{2^{m}\left(2^{j}-1\right)} .
\end{aligned}
$$

We claim next that

$$
\sum_{k=1}^{m} \frac{2^{k}}{k^{2}} \leq 5 \frac{2^{m}}{m^{2}}
$$


for all $m \geq 1$. This inequality may be checked for $m=1,2,3,4$, and follows by induction for $m \geq 5$ using the inequality $(m-1)^{2} \geq(16 / 25) m^{2}$. The inequality from the previous paragraph then becomes

$$
\max _{I} f-\min _{I} f \geq \frac{\pi}{2(m+j)^{2}}-\frac{5 \pi}{\left(2^{j}-1\right) m^{2}} .
$$

Let $j=10$ and assume $m \geq 2$; then

$$
\begin{aligned}
\max _{I} f-\min _{I} f & \geq \frac{\pi}{2(m+10)^{2}}-\frac{5 \pi}{\left(2^{10}-1\right) m^{2}} \\
& \geq \frac{\pi}{2(6 m)^{2}}-\frac{\pi}{200 m^{2}}=\frac{2 \pi}{225 m^{2}}
\end{aligned}
$$

Finally, if $I \subset[0,1]$ has arbitrary length $\varepsilon \leq 1 / 2$, choose $m \geq 2$ such that $2^{1-m} \geq \varepsilon>2^{-m}$. Then for any closed subinterval $J \subset I$ with length $2^{-m}$,

$$
\max _{I} f-\min _{I} f \geq \max _{J} f-\min _{J} f \geq \frac{2 \pi}{225 m^{2}} \geq \frac{\pi}{450(m-1)^{2}} \geq \frac{(\log 2)^{2} \pi}{450(\log \varepsilon)^{2}},
$$

and (2) is established with $c=(\log 2)^{2} \pi / 450$.

\section{MAIN RESUlt}

We say that a function $f \in C[0,1]$ is $M$-Lipschitz at a point $x \in[0,1]$ if there exists a constant $M$ such that for all $y \in[0,1]$,

$$
|f(y)-f(x)| \leq M|y-x| \text {. }
$$

We say that $f$ is Lipschitz at $x$ if it is $M$-Lipschitz for some $M$.

Notice that the set of nowhere Lipschitz functions is the intersection over positive integers $M$ of the set of nowhere $M$-Lipschitz functions. For each $M$, the latter set can be shown to be open (whence the nowhere Lipschitz functions form a Borel set) and dense (whence the nowhere Lipschitz functions are topologically generic) in $C[0,1]$. The prevalence of the set of nowhere Lipschitz functions, as well as Theorem 1 and Proposition 1 , follows from the next proposition.

Proposition 2. There exist functions $g$ and $h$ in $C[0,1]$ such that for all $f \in$ $C[0,1]$, the function $f+\lambda g+\mu h$ is nowhere Lipschitz for Lebesgue almost every $(\lambda, \mu) \in \mathbb{R}^{2}$.

Proof. Let $g$ and $h$ be defined as in the previous section (by (1)), and let $f$ be a function in $C[0,1]$. We wish to show that the set

$$
S=\left\{(\alpha, \beta) \in \mathbb{R}^{2}: f+\alpha g+\beta h \text { is Lipschitz at some } x \in[0,1]\right\}
$$

is a Lebesgue measure zero subset of $\mathbb{R}^{2}$. Let

$$
S_{M}=\{(\alpha, \beta) \in S: f+\alpha g+\beta h \text { is } M \text {-Lipschitz at some } x \in[0,1]\} .
$$

Then $S$ is the union over positive integers $M$ of $S_{M}$; hence it suffices to prove that every $S_{M}$ has measure zero.

Let $N \geq 2$ be an integer, and let us cover $[0,1]$ with $N$ closed intervals of length $\varepsilon=1 / N$. Let $I$ be one of these intervals, and let

$$
J=\left\{(\alpha, \beta) \in S_{M}: f+\alpha g+\beta h \text { is } M \text {-Lipschitz at some } x \in I\right\} .
$$


We will show that $J$ is contained in a disk of radius $C \varepsilon(\log \varepsilon)^{2}$ for some constant $C$ independent of $I$ and $\varepsilon$. It then follows that $S_{M}$ can be covered by $N=\varepsilon^{-1}$ such disks whose total area is $\pi C^{2} \varepsilon(\log \varepsilon)^{4}$. As $\varepsilon \rightarrow 0$, the total area of the cover goes to zero, and therefore $S_{M}$ has measure zero.

Consider two points $\left(\alpha_{1}, \beta_{1}\right)$ and $\left(\alpha_{2}, \beta_{2}\right)$ in $J$. For $i=1,2$, let $f_{i}=$ $f+\alpha_{i} g+\beta_{i} h$ and let $x_{i}$ be a point in $I$ at which $f_{i}$ is $M$-Lipschitz. Then for all $x \in I$,

It follows that

$$
\left|f_{i}(x)-f_{i}\left(x_{i}\right)\right| \leq M\left|x-x_{i}\right| \leq M \varepsilon .
$$

$$
\left|f_{1}(x)-f_{2}(x)-\left(f_{1}\left(x_{1}\right)-f_{2}\left(x_{2}\right)\right)\right| \leq 2 M \varepsilon,
$$

for all $x \in I$, and hence

$$
\max _{I}\left(f_{1}-f_{2}\right)-\min _{I}\left(f_{1}-f_{2}\right) \leq 4 M \varepsilon .
$$

Now $f_{1}-f_{2}=\left(\alpha_{1}-\alpha_{2}\right) g+\left(\beta_{1}-\beta_{2}\right) h$, and thus by Lemma 1 ,

$$
\sqrt{\left(\alpha_{1}-\alpha_{2}\right)^{2}+\left(\beta_{1}-\beta_{2}\right)^{2}} \leq \frac{4 M}{c} \varepsilon(\log \varepsilon)^{2} .
$$

Therefore $J$ lies within a disk of radius $(4 M / c) \varepsilon(\log \varepsilon)^{2}$ as claimed, and the proof is complete.

\section{OTHER RESUlts}

Given $\gamma \in(0,1)$, we say that a function $f \in C[0,1]$ is "Hölder continuous with exponent $\gamma$ ", or $C^{\gamma}$, at a point $x \in[0,1]$ if there exists a constant $M$ such that for all $y \in[0,1]$,

$$
|f(y)-f(x)| \leq M|y-x|^{\gamma} .
$$

Using the methods in this paper one can prove the following result.

Proposition 3. For each $\gamma \in(0,1)$, almost every function in $C[0,1]$ is nowhere $C^{\gamma}$.

For $\gamma>1 / 2$, the proof of the above proposition is virtually identical to the proof of Proposition 2. (The analogue of the set $J$ is shown to be contained in a ball of radius $C \varepsilon^{\gamma}(\log \varepsilon)^{2}$, and hence the total area of the cover is at most $C^{2} \varepsilon^{2 \gamma-1}(\log \varepsilon)^{4}$.) However, for $\gamma \leq 1 / 2$ a higher-dimensional probe is needed; specifically, the dimension must be greater than $1 / \gamma$. (The necessity of this high a dimension can be proved in much the same way that the necessity of a twodimensional probe was proved at the beginning of $\S 2$. Instead of multiplying by $x$, the basis functions of a hypothetical $n$-dimensional probe are multiplied by the coordinates of a "space-filling" function from $[0,1]$ onto the unit cube in $\mathbb{R}^{n}$; this function can be constructed to be Hölder continuous with exponent $1 / n$. It follows that for $\gamma \leq 1 / n$, there is no $n$-dimensional probe for the set of continuous functions which are nowhere $C^{\gamma}$.)

The main technical difficulty in proving Proposition 3 is constructing a set of $n$ functions for which one can prove an analogue of Lemma 1. One approach is to replace the functions $g$ and $h$ with functions $\left\{g_{1}, g_{2}, \ldots, g_{n}\right\}$ defined by

$$
g_{j}(x)=\sum_{k=1}^{\infty} \frac{1}{k^{2}} \cos 2^{(k-1) n+j} \pi x .
$$


Once the higher-dimensional probe is constructed, the necessary changes to the proof of Proposition 2 are minor.

In [5] it is shown that a countable intersection of prevalent sets must be prevalent. This, together with Proposition 3, implies the following result.

Proposition 4. Almost every function in $C[0,1]$ is nowhere $C^{\gamma}$ for any $\gamma>0$.

With further modification to the probe functions (replacing $1 / k^{2}$ with a decaying exponential), one can further prove the following result. (By $C^{\gamma}[0,1]$ we mean the space of functions which are uniformly $C^{\gamma}$ on $[0,1]$.)

Proposition 5. For $0<\gamma<1$, almost every function in $C^{\gamma}[0,1]$ is nowhere $C^{\delta}$ for any $\delta>\gamma$.

Propositions 4 and 5 are also known to be true in the context of genericity [1].

\section{ACKNOWLEDGMENTS}

The author would like to thank T. Sauer and J. Yorke for encouraging his work on this problem and for their helpful suggestions about this paper, and the referee for many thoughtful comments. Much of this work was done while the author was an ONT Postdoctoral Fellow at the Naval Surface Warfare Center, Silver Spring, Maryland.

\section{REFERENCES}

1. H. Auerbach and S. Banach, Uber die Höldersche Bedingung, Studia Math. 3 (1931), 180184.

2. S. Banach, Über die Baire'sche Kategorie gewisser Funktionenmengen, Studia Math. 3 (1931), 174-179.

3. J. P. R. Christensen, On sets of Haar measure zero in abelian Polish groups, Israel J. Math. 13 (1972), 255-260.

4. G. H. Hardy, Weierstrass's non-differentiable function, Trans. Amer. Math. Soc. 17 (1916), 301-325.

5. B. R. Hunt, T. Sauer, and J. A. Yorke, Prevalence: a translation-invariant "almost every" on infinite-dimensional spaces, Bull. Amer. Math. Soc. (N.S.) 27 (1992), 217-238.

6. __ Prevalence: An addendum, Bull. Amer. Math. Soc. (N.S.) 28 (1993), 306-307.

7. J. L. Kaplan, J. Mallet-Paret, and J. A. Yorke, The Lyapunov dimension of a nowhere differentiable attracting torus, Ergodic Theory Dynamical Systems 4 (1984), 261-281.

8. K. Kuratowski, Topology, Vol. 1, Academic Press, New York, 1966.

9. R. D. Mauldin, The set of continuous nowhere differentiable functions, Pacific J. Math. 83 (1979), 199-205.

10. 121 (1986), 119-120.

11. S. Mazurkiewicz, Sur les fonctions non dérivables, Studia Math. 3 (1931), 92-94.

12. J. C. Oxtoby, Measure and category, Springer-Verlag, New York, 1971.

13. D. L. Renfro, Some supertypical nowhere differentiability results for $C[0,1]$, Doctoral Dissertation, North Carolina State University, 1993.

Institute for Physical Science and Technology, University of Maryland, College Park, Maryland 20742

E-mail address: bhunteglue.umd.edu 